\title{
On the Integration of Sensor Networks and General Purpose IT Infrastructure
}

\author{
Wouter Horré, Sam Michiels, Nelson Matthys, Wouter Joosen, Pierre Verbaeten \\ IBBT-DistriNet Research Group, Department of Computer Science, \\ K.U.Leuven, Celestijnenlaan 200A, B-3001 Leuven, Belgium \\ \{wouter.horre,sam.michiels,nelson.matthys\}@cs.kuleuven.be
}

\begin{abstract}
Integrating sensor networks with general purpose IT infrastructure has been confirmed as a complex problem in the development and management of industrial sensor applications. This integration is complex because a single general purpose application queries multiple sensor networks and a single sensor network has to serve multiple applications. This paper identifies three challenges that need to be addressed by middleware in order to enable this integration: federated management, interoperability and adaptability across the whole infrastructure. The paper presents a taxonomy of middleware solutions and positions the identified challenges in a survey of state-of-the-art middleware research we conducted earlier. By looking at sensor network middleware from an application (business) perspective, we come to the conclusion that time may have come to consolidate middleware efforts presented during the past years.
\end{abstract}

\section{Categories and Subject Descriptors}

C.2.4 [Computer-Communication Networks]: Distributed Systems-Distributed Applications; A.1 [General Literature]: [Introductory and Survey]

\section{General Terms}

Design, Management

\section{Keywords}

Sensor networks, middleware, integration

\section{INTRODUCTION}

Early sensor network deployments typically consisted of an isolated sensor network running one application and sending its data to a single sink node. This setting works well for scientific data collection and similar applications, but limits the usability of sensor networks in more complex scenarios. These scenarios, such as in an industrial environment, use

Copyright ACM, 2007. This is the author's version of the work. It is posted here by permission of ACM for your personal use. Not for redistribution. The definitive version was published in Proceedings of the 2nd International Workshop on Middleware for Sensor Networks (MidSens'07), http://doi.acm.org/10.1145/1376860.1376862 complex deployments where multiple sensor networks are interconnected through general purpose networks, allowing applications to use several sensor networks and sensor networks to serve multiple applications.

Figure 1 shows an example of such a complex sensor network deployment. The sensor networks on the left are situated in the storage facilities of a harbor. They are formed on-the-fly by (resource-constrained) sensor nodes attached to containers stored inside and supported by permanently deployed (more powerful) devices. These permanent devices are interconnected and form a network of gateways (blue) towards the Internet (red). The right hand side shows sensor networks deployed on boats and trains. In this case, the gateway devices communicate using long-range technologies, such as GPRS, UMTS or satellite. Various general purpose devices (application servers, laptops, PDAs, smartphones, ...) in the back-end (green) interact with the sensor networks through the Internet. PDAs and smartphones might also interact directly with the sensor networks whenever they come within communication range.

A broad range of applications can be deployed on this heterogeneous infrastructure, possibly at the same time. For example, the transport planning application of a logistics provider running on an application server interacts with the various sensor networks to gather the up-to-date location estimates it needs to optimize the allocation of containers to e.g. trucks. Customs officers in the port interact with the sensor nodes through their PDAs to identify the containers and products in their vicinity. Technicians receive an alarm whenever the temperature in a cooled container raises above a threshold to enable rapid interventions that prevent the goods in these containers from becoming worthless.

Such complex scenarios increase the importance of some existing challenges and introduce new ones. Support for heterogeneity, scalability and security has been important for a long time and becomes even more important with the integration of sensor networks and general purpose IT infrastructure. This integration, however introduces also novel challenges that are often not relevant within the borders of a sensor network. Middleware support is desirable to support the application developers and network administrators in handling these challenges.

The contribution of this paper is twofold: (1) we show that current state-of-the-art middleware support for integration of sensor networks and general purpose IT infrastructure is premature and (2) we identify the new challenges for middleware introduced by this integration. The remainder of this paper is structured as follows: Section 2 illustrates the need 


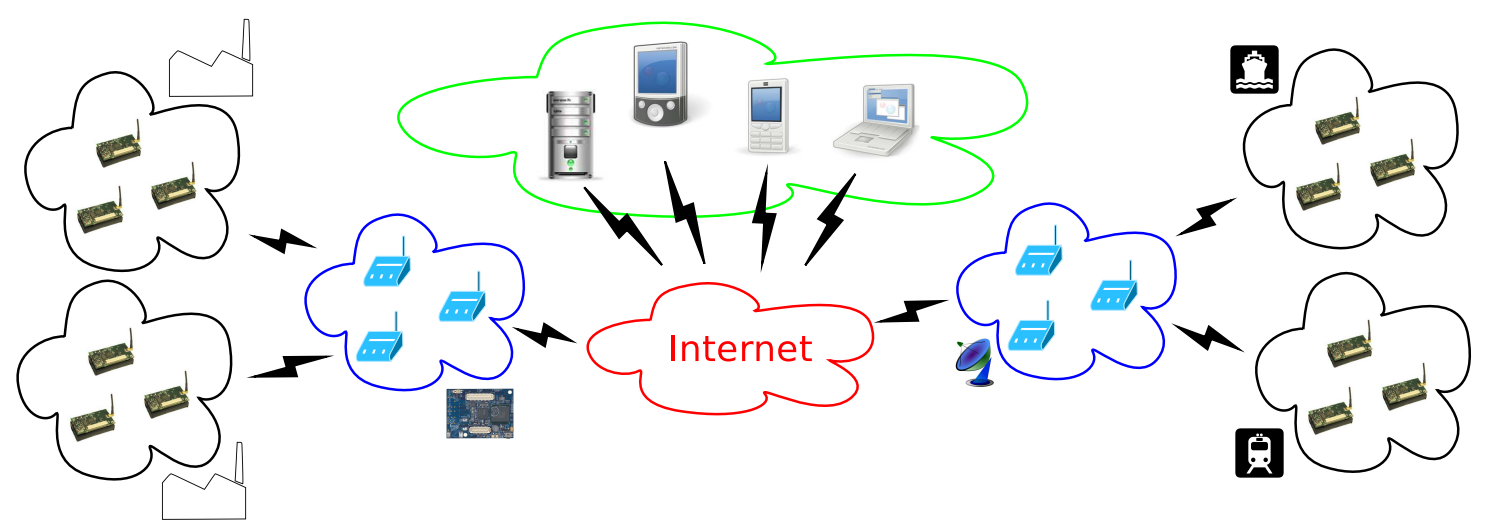

Figure 1: A complex sensor network deployment: multiple sensor networks (black) are connected to various general purpose devices (green) via a network of gateways (blue) and the Internet (red).

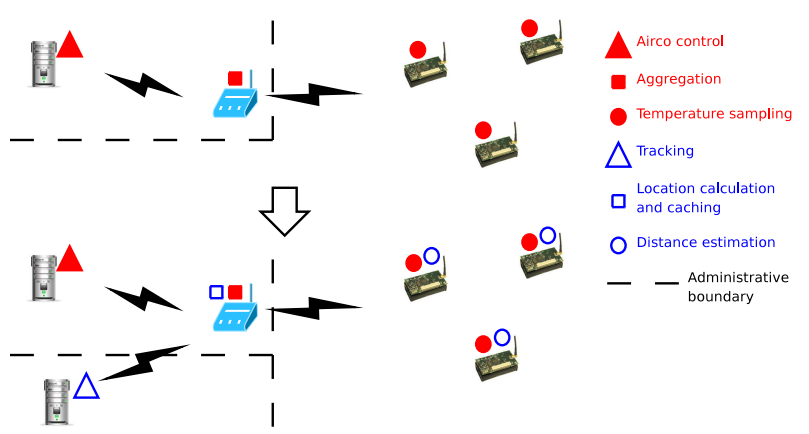

Figure 2: Illustration of the need for integration: deployment of a new back-end application requires changes on the gateway and in the sensor network, even crossing administrative boundaries.

for an integrated approach for sensor network middleware. Section 3 discusses the taxonomy and the conclusions that emerged from a survey of state-of-the-art sensor network middleware we conducted earlier. In Section 4 we identify the challenges introduced by the integration of sensor networks and general purpose infrastructure. Section 5 contains some thoughts on how to tackle these challenges. Finally, we conclude this paper in Section 6 .

\section{THE NEED FOR AN INTEGRATED AP- PROACH}

We argue that integration in the domain of middleware for sensor networks would be beneficial. Instead of zooming in on just one aspect, an integrated approach sets a step back and takes a wide angle view at the whole problem. It tries to harmonize the approaches tackling the different subproblems and addresses issues that crosscut them. To support our claim that integration is needed, we present an example scenario that would definitely benefit from an integrated approach. The example shows how a new backend application requires also changes on the gateway and in the sensor network.

Figure 2 shows the sensor network in one of the storage facilities from Figure 1 at two moments in time. The upper part of the figure shows the situation with a single application using the sensor network to control the air conditioning. The sensor nodes periodically sample their temperature sensor and send this measurement to the gateway tier. The gateway aggregates the values and sends an alarm to the facility management application whenever the average temperature increases above a given threshold.

The infrastructure on which this application runs is not completely controlled by the owner of the storage facility. Only the back-end server and the gateway(s) are under full control of the network administrator employed by the storage facility owner. The sensor nodes attached to the containers stored inside the storage facility are owned by the respective owners of the containers.

The lower part of Figure 2 shows the situation after the deployment of software components needed for tracking containers by their owner(s). To enable tracking of the containers by their owner, localization of the nodes must be available. This requires deployment of a distance measuring component in the sensor network and a location calculation service on the gateway. To enhance the performance and the life time of the sensor network, the administrator also decided to deploy a location caching component on the gateway.

Deployment of a tracking application requires installation of components in the back-end, on the gateway and in the sensor network, crossing administrative boundaries. Manually installing these components will be time consuming and error-prone, thus support for automating some parts of this process is desirable. However, since the changes are to be made across back-end, gateway and sensor network, such support will require an integrated approach along these parts of the infrastructure.

This example is only one out of the many scenarios that would benefit from an integrated approach. Another example is the management of the quality of service (QoS) delivered by a sensor network in the presence of multiple client applications. This management includes selecting the appropriate set of nodes to answer a particular request, introducing caching to improve the sensor network life time, negotiation with back-end applications on the required quality, etc. 


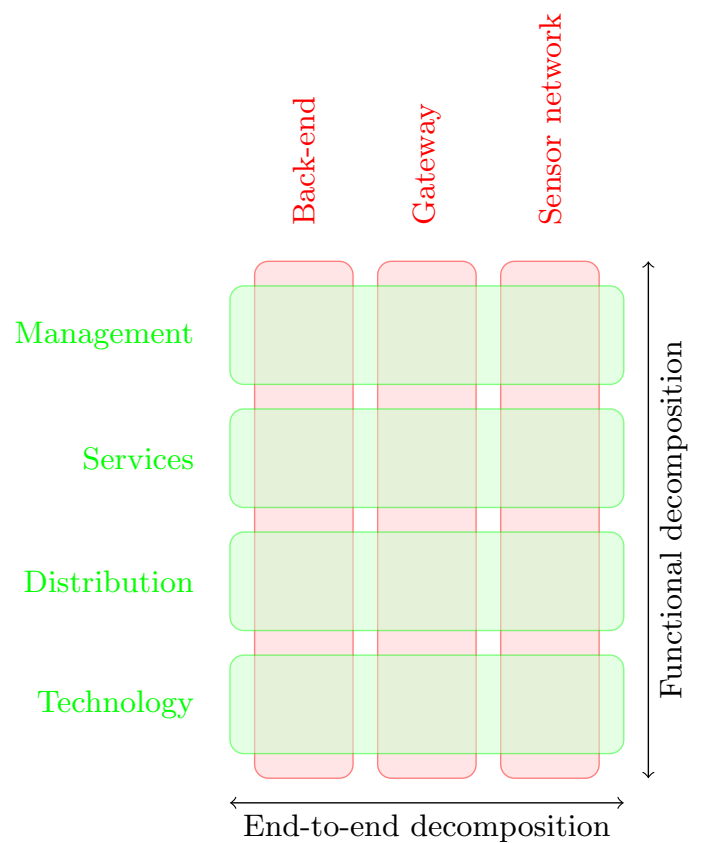

Figure 3: A two-dimensional taxonomy for sensor network middleware

\section{TAXONOMY}

We conducted a survey of middleware in the sensor network domain to support our search for integrated approaches. During our work we developed a two-dimensional taxonomy to classify state-of-the art middleware. In this paper we first present this taxonomy and then discuss the main conclusions that can be drawn from our survey. We refer the interested reader to [9] for the complete survey.

Our taxonomy (Figure 3) classifies middleware along twodimensions: (1) an end-to-end decomposition and (2) a functional decomposition. The first dimension is a decomposition into the different parts that can be identified in an end-to-end integrated infrastructure; the second dimension highlights key functionalities of the middleware.

\subsection{End-to-end decomposition}

The first dimension of our taxonomy classifies a middleware approach according to which tier in the end-to-end spectrum it targets. This dimension consists of three tiers: the sensor network, the gateway and the back-end. The three tiers are discussed in some more detail below. Both the types of hardware and the functionality typically found in the tier are discussed.

Sensor network The sensor network tier contains the highly resource constrained sensor and actuator nodes. The functionality typically found in this tier includes sensor sampling, local data filtering and aggregation, tracking, localization, etc.

Gateway The range of devices that fulfill the gateway functionality is quite broad. In simple scenarios, it is typically a single device communicating with a base station sensor node via serial communication. In complex scenarios it is more likely to be an overlay of more power- ful mote-like devices (such as Intel Mote 2 or Stargate 2) or perhaps multiple tiers of devices which capabilities gradually increase towards the capabilities typically found in general purpose hardware. The gateway functionality includes providing communication with general purpose devices, complex multi-node data aggregation and fusion, authorization, caching, etc.

Back-end The back-end consists of the various general purpose hardware platforms. These platforms are very heterogeneous in their capabilities: ranging from smartphones to enterprise application servers. This tier implements business applications, user consoles, etc.

In some deployments, the border between the tiers can be vague. For example, PDAs and smartphones might be capable of direct communication with sensor nodes in their vicinity, thus bypassing the gateway level. Or the more powerful sensor nodes might be able to communicate with general purpose hardware and as such perform gateway level functionality.

\subsection{Functional decomposition}

Along this dimension we decompose middleware in four conceptual layers of functionality, similar to the layering of middleware introduced in [14]. The categories we identified are: technology, distribution, services and management.

Technology The technology layer hides some of the peculiarities of the underlying operating system and hardware. It provides abstractions that make it easier to program a single node. It may also extend or enhance the functionality provided by the operating system to facilitate the development of the other layers.

Distribution The distribution layer is similar to the technology layer, but aims at distributed programming. It provides abstractions and mechanisms that ease the implementation of distributed programs.

Services Besides local and distributed abstractions, middleware also provides reusable services to the application developers. This further eases the development of applications, since the application developer doesn't have to re-implement this recurrent functionality.

Management The management layer groups all middleware efforts that support co-ordinated management of a (sensor network) deployment. This includes support for starting or stopping services or devices, adding, removing or replacing services, etc. The middleware can support manual management (executing triggers from humans), semi-automated management (some parts are automated, but still human-in-the-loop) or automated management based on e.g. policies or QoS requirements.

It must be noted that this layering is conceptual and does not imply a layered implementation. Especially in the sensor network tier, the opposite is likely to be true. The scarce resources often necessitate an implementation that is not strictly layered. For example, services can be implemented directly on top of the technology layer without using the abstractions provided by the distribution layer or a system in the distribution layer can target one specific operating system and built directly on top of it. 


\subsection{Discussion}

From our survey in [9], it can be seen that a lot of work has already been done in all distinct areas of our taxonomy. However, we argue that integration (see Section 2) is not adequately addressed in the state-of-the-art. Both integration along the end-to-end decomposition dimension (end-to-end integration) and integration along the functional decomposition dimension (cross-layer integration) are not fully supported in current middleware.

\subsubsection{End-to-end integration}

An end-to-end integrated approach takes a broader look on the combination of the sensor network, gateway and backend tiers. It tries to provide a uniform approach across the different tiers. Of course, such an approach can build upon the work already done in the three tiers separately. We believe that the integration of existing work in the different tiers into one overall framework can add significant value and that the integrated whole is worth more than the sum of its parts.

Initial efforts in the direction of an integration across the three tiers can already be found in the literature. The RUNES component model [2] is implemented on devices in the three tiers. Gridkit [4] was originally designed for backend systems but has been customized to also run on gateway and sensor devices [6]. These efforts create the possibility to use the same platform across the different tiers, but work on the actual integration is lacking.

In industry, there are similar observations: the J2ME edition of the Java Technology has been introduced in the sensor network tier by Sun Microsystems. Their Sun SPOT (Small Programmable Object Technology) [13] is a mote class device which runs a J2ME compliant Java virtual machine. The series of Microsoft .NET platforms has been extended with a .NET Micro Framework and Crossbow Technology Inc. is shipping Imote2 devices preloaded with this framework [10].

These efforts are valuable and need to be continued towards a complete end-to-end integration. We believe this integration is a prerequisite for the widespread adoption of sensor networks in complex business applications. Costeffective application development is only feasible if developers can build their applications on top of one platform that allows them to implement functionality in the three tiers.

\subsubsection{Cross-layer integration}

Cross-layer integration is already common in traditional middleware systems. As can be seen from the survey, J2EE and .NET are both active in the four conceptual layers along the functional decomposition axis. This cross-layer integration has two main advantages. First, it makes the middleware as a whole easier to understand, use and maintain. Adopting a cross-layer approach implies structuring the four layers and their interactions in an overall framework. Such a framework is easier to understand than an ad hoc composition of functionality from the four layers. Second, a cross-layer approach makes it easier to address concerns that crosscut the conceptual layers. For example, enforcing security has consequences on the four conceptual layers we identified. Implementing such a concern is a lot easier if the four layers are structured in an overall framework.

In the gateway and sensor network tier, cross-layer integration is not yet mature. There is already some work done, but these efforts have not yet reached the level of maturity found in traditional middleware.

Impala [11] includes work in three of the four conceptual layers. It includes technology for installation and storage of code updates, a code distribution service and an Application Adapter that adapts the running application to the current state of the node and to changes in its environment (e.g. number of direct neighbors, etc.). These various parts are integrated in a middleware architecture. Although Impala has some cross-layer features, these are rather limited. For example, there is only one service (the code distribution) and there is no support for adding others.

TinyCubus [12] consists of three main parts, a data management framework, a configuration engine and a cross-layer framework. The data management framework adapts both system and application components according to certain criteria. The configuration engine provides the technology and the code distribution service needed to support reprogramming of the nodes. The code distribution service uses application knowledge about the sensor topology to optimize its behavior. The sharing of this knowledge is possible through the cross-layer framework. This framework eases cross-layer interactions and provides a state repository to facilitate cross-layer data sharing.

Gridkit [4] uses component frameworks to structure the technology, distribution and services layers. In the technology layer, Gridkit's overlay framework structures the implementation of network overlays. The interaction framework provides a structured approach to distributed interaction styles. Gridkit also includes frameworks for various services, such as resource management and resource discovery. These frameworks are built on top of the reflective component model OpenCOM [3] to enable reasoning over the structure of the system and facilitate fine grained adaptation.

Although the potential benefits of cross-layered approaches for sensor networks have been identified earlier [5, 1], there are only few efforts to tackle cross-layering using a structured approach. Implementing cross-layer interactions in an ad hoc manner may lead to complex, hard to maintain systems. Therefore we believe a solid approach for engineering cross-layered sensor network systems is vital.

\section{CHALLENGES FOR END-TO-END IN- TEGRATION}

The example in Section 2 illustrates the need for an integrated approach across the different parts of the infrastructure. We believe that such an end-to-end integration can provide substantial benefits for the application developers and network administrators in complex sensor network scenarios. However, state-of-the-art middleware doesn't address end-to-end integration sufficiently (Section 3.3).

Realizing this integration is not a trivial task. Several challenges need to be addressed in the middleware. On the one hand, some existing sensor network challenges are strengthened as a consequence of the integration with general purpose IT infrastructure. On the other hand, end-toend integration introduces some novel challenges that are not present within the borders of a single sensor network.

Heterogeneity, scalability and security have long been important concerns in sensor network and general purpose distributed systems research. Integration of sensor networks 
with general purpose IT infrastructure further stresses their importance. The heterogeneity of both domains is now combined, the number of sensor nodes that need to be handled is not limited by the borders of a sensor network anymore and making sensor networks accessible over the Internet exposes them to a lot of new security threats.

In addition to strengthening these existing challenges, the integration of sensor networks and general purpose IT infrastructure introduces some novel challenges that need to be addressed by middleware:

Federated management It was already mentioned in Section 2 that different parts of the infrastructure will probably be owned by different companies. This renders central management and system administration infeasible since the infrastructure spans multiple administrative domains. On the one hand, this decreases the complexity of the management task for the administrators, since each of them only has to manage part of the infrastructure. On the other hand, since these systems are connected and depend on each other, complete autonomous management of the subsystems is not desirable. Some kind of federated management will be inevitable, therefore middleware must support this kind of distributed management.

Interoperability An infrastructure that spans multiple administrative domains will inevitably contain hardware and software platforms from multiple vendors because each company can autonomously decide which platform it deploys. The gateway infrastructure, for example, is deployed for the port authorities by vendor A. The containers have a sensor node of vendor X, Y or $\mathrm{Z}$ depending on their manufacturer. Several other platforms are deployed in the back-end by the users of the sensor network infrastructure: logistics providers, customs authorities, etc. For the whole infrastructure to be useful, these platforms have to be able to co-operate. Interoperability is therefore an important challenge.

End-to-end adaptability The integration of sensor networks with general purpose IT infrastructure introduces sources of dynamism not present within the borders of a sensor network. An example is the scenario presented in Section 2: sensor networks are deployed as reusable infrastructure and thus have to adapt to changes in the number of users and their requirements. Middleware support for this adaptability across the back-end, gateway and sensor networks tiers is desirable. The sources of dynamism that trigger these endto-end adaptations are present in addition to the ones present within the borders of a sensor network (such as intermittent network connections, device failures, environmental changes, etc.)

Most of the challenges introduced by the end-to-end integration have impact on multiple or all of the conceptual layers along the functional decomposition axis of our taxonomy (Section 3). Interoperability, for example, is needed on the four layers to be useful. Interoperability on the technology and distribution layers is nice, but useless if the services that operate on top of them are unable to co-operate because of incompatibilities.
Thus, although we focused on end-to-end integration in this paper, cross-layer integration is equally important. The success of end-to-end integration depends on the degree of cross-layer integration. The challenges discussed in this paper are introduced by end-to-end integration, but can also serve as driving challenges for research on cross-layer integration.

\section{TOWARDS AN END-TO-END INTEGRA- TION}

Looking at the state-of-the-art, we see good solutions in the three tiers of our taxonomy. These solutions are however mostly isolated from each other and are often unable to cooperate. We argue that time may have come to consolidate these efforts and combine them in an integrated approach.

Other domains have been dealing with integration problems with similar challenges as the ones identified in Section 4. Therefore we believe it is useful to have an inspiring look at the solutions that have been developed in these domains.

In the domain of enterprise integration, several integration patterns [8] have been developed. The use of a messaging bus is one of these patterns, one of its best-known incarnations being the enterprise service bus (ESB) [15]. Mediation is an important concept in ESBs; mediators alter the content of the messages on the bus, for example, to translate a message to a different protocol (transcoders).

The bus concept, in particular the use of mediators, might be inspiring for the development of similar techniques in the integrated approach we envision. It can provide interoperability through mediators of the transcoder type that translate between protocols used in various parts of the infrastructure.

On top of this interoperable bus, federated management and end-to-end adaptability might be implemented using a mechanism similar to the one used by MiLAN [7]. MiLAN is a quality management solution for sensor networks. It decouples the process of making the decision of which sensors to enable from the actual execution of this decision. This decoupling is realized by defining a set of generic MiLAN commands that are translated to network specific operations by network plug-ins.

To support federated management and end-to-end adaptability, one can develop a generic format to express requests for management actions (e.g. enable or disable a sensor) or adaptations (e.g. install new functionality). These generic requests would then be interpreted and transformed by a mediator provided by the network administrators.

For the example scenario in Section 2, one could provide a generic operation install. The tool that deploys the tracking application on the application server can then send an install localization command to the gateway over the bus. When the management component on the gateway receives this command, it will evaluate this request and perform the necessary action. In this case, it will send an install distance measuring command to the sensor nodes, install a location calculation component and additionally install a caching component to comply with the local policy.

What operations should be included in a complete set of generic commands is an open issue. Also the management components should preserve the consistency of the system and co-operate to resolve conflicts between the policies in 
the different administrative domains.

\section{CONCLUSION}

In this paper, we have argued for an integrated middleware approach to tackle the integration of sensor networks and general purpose IT infrastructure. First, we have illustrated the need for such an integrated approach with a scenario where this would bring substantial benefits to the application developers and network administrators.

We have presented the results of an extensive survey of state-of-the-art middleware we conducted earlier [9]. We discussed the two-dimensional taxonomy that emerged during our work on the survey. The first dimension of this taxonomy divides the complex infrastructure in three tiers (sensor network, gateway and back-end) and we classify a middleware approach along this dimension according to the tier it targets. The second dimension identifies four groups of functionality provided by middleware: technology, distribution, services and management.

From the survey it can be concluded that state-of-theart middleware often focuses on one category in our taxonomy. However, support for integration of the four conceptual layers along the functional decomposition dimension (crosslayer integration) is only available in the back-end tier. Support for the integration of the three tiers (end-to-end integration) is almost completely missing.

Subsequently, we identified the challenges introduced by the integration of sensor networks with general purpose IT infrastructure. We also highlighted that these challenges, although introduced by end-to-end integration, can also be motivating challenges for research on cross-layer integration.

Finally, we have argued that an integrated approach tackling these challenges should consolidate existing solutions from the three tiers and pointed out that solutions for similar problems in other domains can be inspiring. We proposed the concept of a message bus as used in enterprise integration as one such inspiring solution.

\section{ACKNOWLEDGEMENTS}

Wouter Horré is a Ph.D. fellow of the Research Foundation - Flanders (FWO).

\section{REFERENCES}

[1] Ian F. Akyildiz and Ismail H. Kasimoglu. Wireless sensor and actor networks: research challenges. $\mathrm{Ad}$ Hoc Networks, 2(4):351-367, October 2004.

[2] Paolo Costa, Geoff Coulson, Richard Gold, Manish Lad, Cecilia Mascolo, Luca Mottola, Gian Pietro Picco, Thirunavukkarasu Sivaharan, Nirmal Weerasinghe, and Stefanos Zachariadis. The runes middleware for networked embedded systems and its application in a disaster management scenario. In Proceedings of the 5th IEEE International Conference on Pervasive Computing and Communications (PERCOM07), March 2007.

[3] Geoff Coulson, Gordon S. Blair, Paul Grace, Ackbar Joolia, Kevin Lee, and Jo Ueyama. A component model for building systems software. In Proceedings of IASTED Software Engineering and Applications (SEA'04), Cambridge, MA, USA, November 2004. ACTA Press.
[4] Geoff Coulson, Paul Grace, Gordon Blair, Wei Cai, Chris Cooper, David Duce, Laurent Mathy, Wai Kit Yeung, Barry Porter, Musbah Sagar, and Wei Li. A component-based middleware framework for configurable and reconfigurable grid computing. Concurrency and Computation: Practice and Experience, 18(8):865-874, July 2006.

[5] Andrea J. Goldsmith and Stephen B. Wicker. Design challenges for energy-constrained ad hoc wireless networks. IEEE Wireless Communications, 9(4):8-27, August 2002.

[6] Paul Grace, Geoff Coulson, Gordon Blair, Barry Porter, and Danny Hughes. Dynamic reconfiguration in sensor middleware. In Proceedings of the First International Workshop on Middleware for Sensor Networks (MidSens), New York, NY, USA, November 2006. ACM Press.

[7] Wendi B. Heinzelman, Amy L. Murphy, Hervaldo S. Carvalho, and Mark A. Perillo. Middleware to support sensor network applications. IEEE Network, 18(1):6-14, 2004.

[8] Gregor Hohpe and Bobby Woolf. Enterprise Integration Patterns: Designing, Building, and Deploying Messaging Solutions. Addison-Wesley Longman Publishing Co., Inc., Boston, MA, USA, 2003.

[9] Wouter Horré, Nelson Matthys, Sam Michiels, Wouter Joosen, and Pierre Verbaeten. A survey of middleware for wireless sensor networks. Technical Report CW 498, Department of Computer Science, K.U.Leuven, August 2007.

[10] Crossbow Technology Inc. Wsn imote2 .builder kit. http://www.xbow.com/Products/productdetails.aspx?sid=267, July 2007.

[11] Ting Liu and Margaret Martonosi. Impala: a middleware system for managing autonomic, parallel sensor systems. In PPoPP '03: Proceedings of the ninth ACM SIGPLAN symposium on Principles and practice of parallel programming, pages 107-118, New York, NY, USA, 2003. ACM Press.

[12] Pedro José Marrón, Andreas Lachenmann, Daniel Minder, Jörg Hähner, Robert Sauter, and Kurt Rothermel. TinyCubus: A flexible and adaptive framework for sensor networks. In Proceedings of the Second European Workshop on Wireless Sensor Networks (EWSN), pages 278-289, January 2005.

[13] Sun Microsystems. Sun SPOT world. http://www.sunspotworld.com/, July 2007.

[14] Douglas C. Schmidt. Middleware for real-time and embedded systems. Communications of the ACM, 45(6):43-48, 2002.

[15] Marc-Thomas Schmidt, Beth Hutchison, Peter Lambros, and Rob Phippen. The enterprise service bus: Making service-oriented architecture real. IBM Systems Journal, 44(4):781-797, 2005. 